Peter Franzkowiak Hermann Schlömer

\section{Entwicklung der Suchtprävention in Deutschland: Konzepte und Praxis}

\author{
Development of Primary Prevention of Addiction in Germany: Conceptions and \\ Practice
}

\section{Zusammenfassung}

Die Phasen der konzeptionellen und praktischen Entwicklung der Suchtprävention in Deutschland von Ende der 60er-Jahre bis heute werden nachgezeichnet. Die aktuelle Suchtprävention ist durch vier integrierte Ansätze geprägt: Förderung von Risikokompetenz in Verbindung mit lebensweltnaher Drogenerziehung; Förderung von Persönlichkeitsentwicklung und Basisfähigkeiten der Lebensbewältigung; Ermöglichung erlebnisorientierter Alternativen; Früherkennung von Entwicklungsproblemen und frühzeitige gezielte Hilfen bei problematischem Drogenkonsum. Zu jedem dieser Ansätze werden musterhafte, z.T. bereits evaluierte Praxisbeispiele vorgestellt. Abschließend werden offene Fragen und Probleme (selbst-)kritisch beleuchtet.

\section{Schluisselwörter}

Drogenerziehung · Suchtprävention · Lebenskompetenzförderung • Risikokompetenz · Schadensminimierung

\section{Abstract}

The conceptions and practice of drug education and primary prevention of addiction in Germany since the late $1960 \mathrm{~s}$ will be described. At the present stage, four major conceptions are relevant: the promotion of risk-taking competence, combined with setting-oriented and life style-sensitive drug education; the promotion of life skills and competencies for constructive coping of problems; experiences of adventure-oriented alternatives to drug use; establishment of early screening of developmental hazards and problems as well as early support with regard to problematic drug use. All of these conceptions are illustrated by models of good practice, many of them evaluated. Final conclusions shed a (self-)critical light on today still unanswered questions and problems of primary addiction of prevention.

Key words

Drug education $\cdot$ primary prevention of addiction $\cdot$ promotion of life skills $\cdot$ risk-taking competence $\cdot$ harm reduction

\section{Zum Wandel der Präventionsansätze}

Ungeachtet der weit verbreiteten fachlichen Kritik an der Kampagne „Keine Macht den Drogen“ ist diese, nach Einstellung durch das Bundesgesundheitsministerium Ende der 90er-Jahre, mit Unterstützung des Bundesinnenministers wieder aufgelegt worden. Ist die Suchtprävention auf dem Stand der 80er-Jahre stehen geblieben? Der Schein trügt. Auch wenn fast alle bisherigen Ansätze in irgendeiner Form in der Praxis noch vorhanden sind - die Ziele, Inhalte und methodischen Ansätze der Suchtprä- vention in der Bundesrepublik Deutschland haben sich seit ihren Anfängen mit dem Kenntnisstand der Wissenschaft, den Erfahrungen der Praxis und der gesellschaftspolitischen Entwicklung verändert.

\section{Drogenprävention durch Abschreckung und Kriminalisierung}

In den späten 60er-Jahren begannen mehr und mehr Jungerwachsene und Jugendliche, Haschisch und LSD zu konsumieren. Heroin erschien auf dem Drogenmarkt. Die ersten verelendeten 
Opiatabhängigen wurden auffällig, die ersten „Drogentoten“ gezählt. Die Sorge, Jugendliche könnten zum Probieren von Haschisch verführt werden und dadurch später ins Elend der Heroinabhängigkeit abrutschen, griff um sich. Der Wunsch, den Heranwachsenden dieses Schicksal zu ersparen, bestimmte die Konzepte drogenpräventiver Aktivitäten in den 70er- und auch noch 80er-Jahren. Dabei ging es im Wesentlichen um die Verhütung jeglichen Konsums illegaler Drogen [1, 2].

In der Praxis der Drogenprävention setzte man auf Abschreckung durch einseitige drastische Darstellungen der Risiken des verbotenen Drogenkonsums. Die bevorzugten stoffkundlichen Belehrungen arbeiteten mit zum Teil unsachlichen Botschaften. Besonders Plakate, Broschüren und andere Materialien von Zoll, Polizei und Krankenkassen aus dieser Zeit waren davon geprägt. Auch viele LehrerInnen, die mit ihren Schulklassen das Buch „Christiane F. - Wir Kinder vom Bahnhof Zoo“ lasen und dessen Verfilmung sahen, taten dies in der Hoffnung auf abschreckende Effekte. Die Strafverfolgung der KonsumentInnen illegaler Drogen, ihre pauschale Verurteilung und Stigmatisierung gehörten mit zur Strategie der Drogenprävention. Die Glaubwürdigkeit der Drogenaufklärer litt erheblich unter den einseitigen Risikodarstellungen und der damit einhergehenden Doppelmoral. Die überzogenen Darstellungen machten viele Adressaten eher neugierig. Die erhofften Erfolge blieben aus.

\section{Von der abstinenzorientierten Drogenerziehung zur ursachenorientierten Suchtprävention}

Etwa ab Mitte der 70er-Jahre begannen die Fachkräfte der Drogenprävention vermehrt damit, sachlicher über die Risiken der Drogen aufzuklären und an die Vernunft zu appellieren. Das geschah weiterhin hauptsächlich mit dem Ziel, junge Menschen zu völliger Abstinenz in Bezug auf illegale Drogen zu bewegen, sie aber auch vor missbräuchlichem Konsum sog. Alltagsdrogen zu bewahren. Auch das führte nicht zum gewünschten Erfolg, da die Vermittlung kognitiven Wissens als Faktor der Verhaltensbeeinflussung überschätzt wurde. Hinzu kommt, dass Aufklärung mit Negativzielen und Furchtappellen oft nicht fruchtet [3].

Vor diesem Erfahrungshintergrund richtete sich die Aufmerksamkeit ab etwa Anfang der 80er-Jahre stärker auf die individuellen und sozialen Ursachen von Suchtproblemen sowie die Motive des Drogenkonsums. Sucht und nicht so sehr Drogenkonsum wurde als wesentliches Problem, das es zu verhindern galt, aufgefasst. Konsequenterweise kamen nun auch die Abhängigkeit von Alkohol, Tabak und Medikamenten sowie stoffungebundene Verhaltenssüchte wie z.B. Spielsucht oder Essstörungen mit in den Blick.

\section{Die Hinwendung der Suchtprävention zur Gesundheitsförderung}

Erkenntnisse der Entwicklungspsychologie, der Copingforschung und der Gesundheitswissenschaften, die 1986 in der Ottawa-Charta der WHO (Weltgesundheitsorganisation der Vereinten Nationen) ihren Niederschlag fanden, leiteten in der Suchtprävention gegen Ende der 80er-Jahre zwar nicht den gänzlichen Abschied von der Ursachenorientierung, aber einen Wechsel von einer eher krankheitsfixierten zu einer mehr ge- sundheitsbezogenen (salutogenetischen) Perspektive ein. Dementsprechende suchtpräventive Konzepte orientierten sich stärker an den Bedingungen, Kompetenzen und Faktoren, die zur Gesundheit befähigen, die Gesundheit schützen und zur Unverletzlichkeit bzw. Immunisierung gegenüber Gesundheitsstörungen und Suchtgefährdungen beitragen [4-6].

Viele der für Suchtprävention Verantwortlichen wendeten sich zunächst von der Aufklärung über Drogen ab. Suchtprävention ging oft suchtmittelunspezifisch vor und konzentrierte sich darauf, grundlegende Lebenskompetenzen („life skills“) als Schutzfaktoren gegenüber den vermuteten Ursachen von Suchtproblemen zu entwickeln und zu stärken. Zielgruppen dafür waren nicht mehr nur Jugendliche, sondern auch Kinder, um möglichst frühzeitig in Familien und Erziehungsinstitutionen gegenüber Suchtgefahren zu immunisieren. Nicht von ungefähr nahmen viele suchtpräventive Fachkräfte in der Bundesrepublik Deutschland die Plakate der Suchtpräventionsstelle der Stadt Zürich aus der Serie „Sucht hat viele Ursachen“ mit ihren positiven Botschaften wie „Spielen ist nicht nur für Kinder Musik in den Ohren - Gemeinsam spielen. Wir werben dafür" begeistert auf. Typisch für diese Phase war auch die Geburt der Kampagne „Kinder stark machen“. Nur der auf den Kampagnenplakaten der Bundeszentrale für gesundheitliche Aufklärung (BZgA) verwendete Halbsatz „Schlimm genug, dass es Drogen gibt ..." erinnert noch an die Phase der Drogenprävention.

Quantitative und qualitative Studien in den USA und der Bundesrepublik Deutschland aus den 70er- und 80er-Jahren [7-11] belegten eine Funktionalität des Drogenkonsums im Hinblick auf die Bewältigung von Entwicklungsaufgaben des Jugendalters. Das motivierte PädagogInnen, funktionelle Äquivalente zum Drogenkonsum anzubieten. Es ging dabei darum, alternative Mittel zur Befriedigung der Bedürfnisse nach sozialer Anerkennung und Statuserwerb, nach Identitäts- und Lebensstilfindung, nach Grenzerfahrungen und Abenteuer zu erschließen. Darüber hinaus verfolgten erlebnis-, medien- und kulturpädagogische Aktivitäten der Suchtprävention das Ziel, Selbstwirksamkeitserfahrungen zu vermitteln, das Selbstbewusstsein der Beteiligten zu stärken, soziale Kompetenzen wie Durchsetzungsvermögen, Konfliktlösungsfähigkeit und Durchhaltevermögen einzuüben.

Bestätigung erfuhr diese konzeptionelle Weiterentwicklung der Suchtprävention Anfang der 90er-Jahre durch die Befunde einer von der BZgA in Auftrag gegebenen „Expertise zur Primärprävention des Substanzmissbrauchs“ [12,13]. Drei zentrale Ergebnisse lauteten:

- Abschreckungsorientierte Informationsvermittlung über psychoaktive Substanzen ist im günstigsten Fall ineffektiv, im ungünstigsten Fall schädlich in Hinblick auf die Verringerung eines Missbrauchsverhaltens.

- Die Förderung von Lebenskompetenzen („life skills“) bei Jugendlichen ist eine wirksame präventive Maßnahme im Hinblick auf die Verhinderung oder Verzögerung des Konsumbeginns und die Verhinderung eines langfristigen Missbrauchsverhaltens. Das beinhaltet z.B. die Förderung von Problemlösungs- und Kommunikationsfähigkeiten, von Selbstsicherheit und Durchsetzungsvermögen. 
- Als Ergänzung zum Konzept der „life skills“ sind Maßnahmen zur Schaffung von Alternativen zum Drogenkonsum positiv zu beurteilen, dies gilt insbesondere für Jugendliche mit hoher sozialer Belastung.

\section{Förderung von Gesundheit und Risikokompetenz}

Die Hinwendung zur Gesundheitsförderung hatte vorübergehend zur Folge, dass Suchtprävention sich immer weniger von allgemeinen Erziehungsaufgaben unterschied. In den 90erJahren setzte sich mehr und mehr die Erkenntnis durch, dass „das Kind nicht mit dem Bade ausgeschüttet“ werden sollte. Insbesondere die Ausbreitung von Ecstasy als so genannter Partydroge öffnete die Augen dafür, dass viele Jugendliche rein zum Spaß, ohne Problemdruck und Protestbedürfnis, aber zugleich ohne genügendes Wissen über die Risiken des Konsums Drogen gebrauchen. Das führte zu einer konzeptionellen Rückbesinnung auf den erzieherischen, verhaltensorientierenden Wert von Drogenaufklärung. Suchtpräventive Programme wurden wieder stärker mit ihren spezifischen Profilen und Anliegen erkennbar $[14,15]$.

Mittlerweile haben immer mehr Konzepte zur Suchtprävention wieder substanz- und verhaltensbezogene Bestandteile. Seit Ende der 90er-Jahre hat sich die Förderung von Risikokompetenz im Umgang mit Drogen in zahlreichen Projekten zu einem neuen konzeptionellen Akzent entwickelt - nicht nur in der Suchtprävention, sondern seit längerem auch in der Drogenund Überlebenshilfe [16 - 18]. Dieser konzeptionellen Umorientierung sind auch die im Juni 2002 von der Drogenbeauftragten der Bundesregierung vorgelegten Eckpunkte für einen neuen Aktionsplan Drogen und Sucht in einigen Teilen verpflichtet. Nach diesem neuen Plan sollen sowohl die Drogen- und Suchtpolitik als auch Suchtprävention und Suchtkrankenhilfe von drei übergeordneten Zielen und acht Teilzielen geleitet werden (Tab.1).

Tab. 1 Eckpunkte für den Aktionsplan Drogen und Sucht, Juni 2002

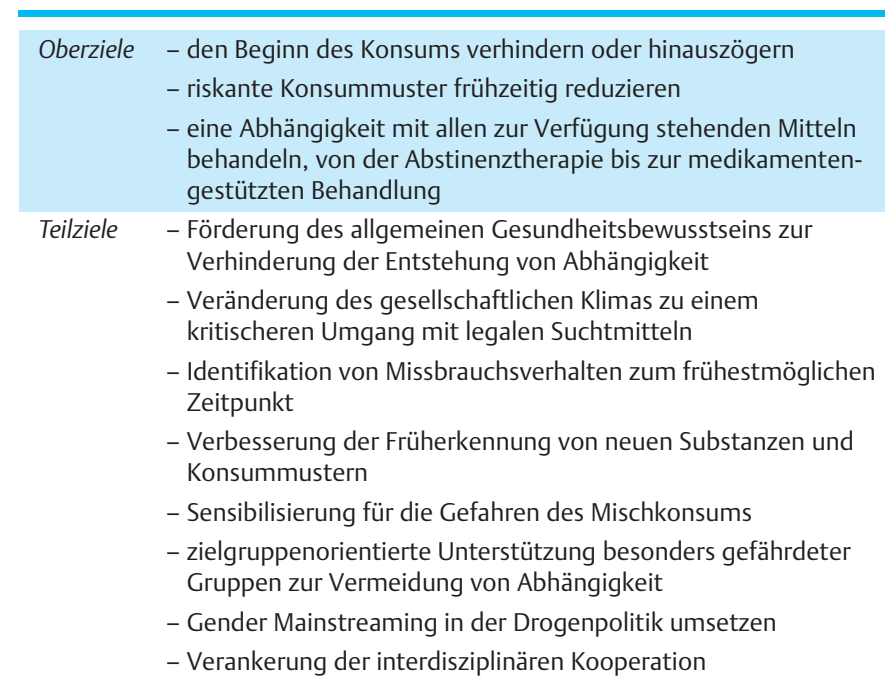

Suchtprävention 2003: Ansätze und Praxisbeispiele

Suchtprävention 2003 besteht aus verschiedenen konzeptionellen und praktischen Komponenten, die im günstigsten Falle alte und neue Leitorientierungen der letzten 30 Jahre organisch miteinander verbinden. Die wichtigsten seien im Folgenden vorgestellt und illustriert. Dabei dokumentiert die Reihenfolge der Darstellung nicht ein Ranking, sondern knüpft nur an dem zuletzt beschriebenen Entwicklungsstadium der Suchtprävention an.

\section{Förderung von Risikokompetenz und Drogenerziehung}

Experimentier- und Risikoverhalten insbesondere von Kindern und Jugendlichen ist aus entwicklungspsychologischer Sicht normal und entspringt sehr oft Bedürfnissen nach sozialer Anerkennung, Genuss, Lust und Abenteuern. Im Umgang mit Risiken erkunden Kinder und Jugendliche ihre Fähigkeiten und Grenzen [19]. Das gilt auch für den Gebrauch psychoaktiver Substanzen, wie die nach wie vor hohe Verbreitung jugendlichen Konsums von Alkohol, Nikotin und Cannabis zeigt. Was die letztgenannte Droge betrifft, haben amerikanische Forscher schon 1990 mit einer bemerkenswerten Längsschnittstudie darauf aufmerksam gemacht, dass der experimentelle Konsum Jugendlicher als relativ normal und zumeist als nicht besonders abträglich für die Gesundheit und Persönlichkeitsentwicklung zu werten ist [20]. In einer Gesellschaft, in der Alkoholkonsum von Erwachsenen geradezu erwartet wird, in verführerischer Weise fast ständig und überall für Alkohol- und Nikotinkonsum geworben wird, der Gebrauch von Psychopharmaka sehr verbreitet und akzeptiert ist, erscheint es plausibel, das „Hineinwachsen in die Drogenkultur der Gesellschaft" als eine für alle Jugendlichen zu lösende Entwicklungsaufgabe zu charakterisieren [11].

Vor diesem Hintergrund liegt es nahe, die Überführung von jugendlichem Risikoverhalten in lebenslange Risikokompetenz in den Zielkatalog der Suchtprävention aufzunehmen. Risikokompetenz umfasst [21-23]:

- informiertes Problembewusstsein über Drogenwirkungen und Risiken des Drogenkonsums;

- kritische Einstellung gegenüber legalen und illegalen Drogen;

- Verzicht auf bestimmte Substanzen (harte Drogen, Selbstmedikation);

- Bereitschaft und Fähigkeit zum konsequenten Konsumverzicht (Punktnüchternheit) in bestimmten Situationen, Lebensräumen und Entwicklungsphasen (Kindheit, frühe Jugend, Schule, Arbeitswelt, Straßenverkehr, Schwangerschaft etc.);

- Vermögen, sich zwischen Abstinenz und mäßigem Konsum in tolerierten Situationen ohne negative Konsequenzen bewusst und verantwortlich entscheiden zu können;

- Entwicklung von Regeln für einen genussorientierten und maßvollen Konsum, Beherrschung von Sicherheitsregeln, die sowohl das persönliche Risiko als auch das für die Umwelt mindern (z.B. kein täglicher Alkoholkonsum).

Mit der Aufnahme der Förderung von Risikokompetenz in den Zielkatalog wird in der Suchtprävention das Realziel „Verhinderung eines längerfristigen Missbrauchsverhaltens" statt der unrealistischen Ausrichtung auf Totalabstinenz handlungsleitend [16, 24-28]. Das beinhaltet sechs Prinzipien und Teilziele (Tab. 2). 
Tab. 2 Prinzipien und Realziele im Risikomanagement

\begin{tabular}{|c|c|}
\hline Immunisierung: & $\begin{array}{l}\text { Aufschub von Konsum- und Probierbeginn bei } \\
\text { legalen Drogen, möglichst lebenslange Abstinenz } \\
\text { gegenüber illegalen Drogen }\end{array}$ \\
\hline Transitionierung: & $\begin{array}{l}\text { Beschränkung des substanzbezogenen Konsums, } \\
\text { aber auch eines Missbrauchs legaler wie illegaler } \\
\text { Substanzen auf einen experimentellen, zeitlich } \\
\text { begrenzten Probierkonsum }\end{array}$ \\
\hline Sensibilisierung: & $\begin{array}{l}\text { Entwicklung von Unterscheidungskriterien } \\
\text { zwischen Genuss, Missbrauch und Abhängigkeit, } \\
\text { Sensibilisierung für riskante und weniger riskante } \\
\text { Konsummuster, Verbesserung von Selbstein- } \\
\text { schätzung und Selbstreflexion }\end{array}$ \\
\hline Lebensweltorientierung: & $\begin{array}{l}\text { Explizite und umfassende, „wahrheitsgemäße“ } \\
\text { und glaubwürdige Aufklärung über Wirkungen, } \\
\text { Nebenwirkungen und Wechselwirkungen des } \\
\text { punktuellen und/oder regelmäßigen Konsums } \\
\text { psychoaktiver Substanzen }\end{array}$ \\
\hline Schadensminimierung: & $\begin{array}{l}\text { Verhinderung von Abhängigkeitsentwicklungen } \\
\text { bei dauerhaftem Missbrauch durch Sicherheits- } \\
\text { regeln, Ermöglichung eines kontrollierten Kon- } \\
\text { sums mit Gefahrenbegrenzung und Notfallhilfe }\end{array}$ \\
\hline $\begin{array}{l}\text { strukturelle Gesundheits- } \\
\text { förderung: }\end{array}$ & $\begin{array}{l}\text { Aufbau und Erhaltung sozialer Unterstützungs- } \\
\text { und Bewältigungsnetzwerke, Einwirkung auf } \\
\text { negative Sozialisations- bzw. Milieubedingungen } \\
\text { von gefährdeten und suchtaffinen Jugendlichen }\end{array}$ \\
\hline
\end{tabular}

Was das Ziel betrifft, den Konsumbeginn zu verzögern, sei hier ein besonders erwähnenswertes und auf andere Drogen durchaus übertragbares Projekt vorgestellt:

\section{Praxisbeispiel: „Be Smart - Don‘t Start“}

Im Rahmen des vom Institut für Therapie- und Gesundheitsforschung in Kiel koordinierten europäischen Wettbewerbs schulischer Nichtraucherförderung verpflichten sich seit sechs Jahren immer mehr Schulklassen, sechs Monate nicht zu rauchen. Wöchentlich bilanzieren und reflektieren sie ihre Erfahrungen, beschäftigen sich in begleitenden Unterrichtsprojekten mit den Motiven und Risiken des Rauchens, analysieren die Versuchungen des Umfelds und der Werbung, entwickeln und üben Möglichkeiten des Neinsagens, erarbeiten und erproben Verhaltensalternativen zum Rauchen [29, 30].

Der Förderung von Risikokompetenz hat Chancen auf Erfolg,

- wenn z.B. Kinder und Jugendliche in der Schule ihre drogenkundlichen Fragen offen stellen, sich sachlich und präzise über Drogen informieren und zu eigenen Standpunkten und Verhaltenssicherheiten finden können;

- $\quad$ wenn in Familien, Schulen und Freizeiteinrichtungen offene Gespräche und selbstkritische Reflexionen über Drogenkonsum und suchtriskantes Verhalten möglich sind. Eine entscheidende Voraussetzung dafür ist ein vertrauensvolles Klima. Hilfreich sind einladende Ansprachen und Situationen (in der Schule auf jeden Fall außerhalb der Leistungsbewertung) sowie eine aufgeschlossene verständnisvolle Haltung gegenüber den jeweils relevanten Konsum- und Verhaltensmotiven. Appelle und moralisierende Reaktionen wirken kontraproduktiv. Benötigt werden GesprächspartnerInnen, die zuhören, sich selbst mit ihren Erfahrungen einbringen, kritische Fragen stellen, nicht ihre Augen verschließen und ggf. Sorgen zum Ausdruck bringen;

- wenn „youth to youth education“ bzw. „peer education“ stattfindet;

- $\quad$ wenn konkrete Trinkregeln und andere Konsumregeln formuliert, verbreitet und zur Diskussion gestellt werden oder wenn Jugendliche durch Verzichtsexperimente gesundheitsbewussteres Konsumverhalten entwickeln. Dafür zwei Praxisbeispiele:

\section{Praxisbeispiel: „Alkohol. Irgendwann ist der Spaß vorbei.“}

Unter diesem Motto startete das Büro für Suchtprävention Hamburg im November 1999 mit Plakaten, Kinospots, Anzeigen und Info-Cards eine Kampagne zur Reduzierung riskanten Alkoholkonsums Jugendlicher im Alter von 15 bis 17 Jahren. Die mittlerweile auch in anderen Bundesländern verwendeten Medien dieser Kampagne thematisieren eines der wichtigsten Motive jugendlichen Alkoholkonsums: die Geselligkeitsförderung. Plakate und Karten erzählen humorvoll und unter Verzicht auf Belehrung Konsumgeschichten, die genussvoll beginnen und mit Peinlichkeiten enden:

- Endlich mal wieder gute Musik. - Alle tanzen. - Alle lächeln mich an. - Alle kreischen mir zu. - Wo ist eigentlich mein Top geblieben?

- $\quad$ Petra kennen gelernt. - Petra zum Lachen gebracht. - Petra abgeknutscht. - Petra in den Ausschnitt gekotzt.

Die Kampagne knüpft so an die Erfahrungen vieler Jugendlicher an und motiviert, über die eigenen Grenzen in Bezug auf Alkoholkonsum nachzudenken. Sie liefert dadurch Orientierungen. An vielen Schulen werden mithilfe der Kampagnenplakate kreative Schreibprojekte durchgeführt, in denen Jugendliche die Geschichten eigener oder miterlebter Konsumerfahrungen z. B. nach dem Muster der Kampagne als Denkanstöße auch für andere aufschreiben.

\section{Praxisbeispiel: „Kontrollierter Konsum durch initiierte} Abstinenz"

Um SchülerInnen die Möglichkeit zu geben, das eigene Verhalten sinnlich zu erfahren, zu hinterfragen und ggf. zu verändern, wird in Schleswig-Holstein bereits seit 1994 dieser gründlich evaluierte Baustein schulischer Suchtprävention durchgeführt. Er besteht im Wesentlichen aus folgenden, aufeinander aufbauenden Modulen:

- Befragung der teilnehmenden Schulklassen in Bezug auf ihr Konsumverhalten

- Vorstellung und Diskussion der Befragungsergebnisse

- Auseinandersetzung mit den Motiven, den erwünschten Wirkungen und unerwünschten Folgen des Konsumverhaltens

- Beschäftigung mit den Entstehungsbedingungen von Suchtproblemen

- $\quad$ kritische Reflexion der eigenen „lieben Gewohnheiten“

- $\quad$ individuelle Vereinbarungen zum vierzehntägigen Verzicht (Reduktion) auf ein Suchtmittel oder auf eine Verhaltensweise wie Fernsehen, Naschen etc. 
Abstinenz-/Reduktionsversuche

- begleitende Besprechungen von alternativen Verhaltensweisen, anderen Hilfsquellen und Schwierigkeiten Auswertung der Erfahrungen

Unwissenheit kann Verhaltensunsicherheiten begünstigen, kann Abstinenzentscheidungen oder die Aneignung von gesundheitszuträglichen Konsumformen erschweren. Die Vermittlung von Wissen über die Wirkungen und Folgen des Gebrauchs legaler und illegalisierter Drogen, so ein weit verbreiteter aktueller Standpunkt, muss daher Bestandteil suchtpräventiver Bemühungen sein. Dabei gilt es, die Fehler der Vergangenheit nicht zu wiederholen. Drogenkundliche Angebote sollten nach heutigem Erkenntnisstand altersgemäß, unaufgeregt und ohne erhobenen Zeigefinger sowie unter Verzicht auf Überdramatisierungen und Bagatellisierungen sachgerecht erfolgen.

Darüber hinaus scheinen sich für die Drogenaufklärung folgende Qualitätsstandards durchzusetzen:

a) zielgruppenspezifisch an den vorhandenen Erfahrungen und Fragen anknüpfen. Diese ergeben sich nicht nur durch eigenen Konsum, sondern auch durch Anschauungen in der Familie, Verwandtschaft, Nachbarschaft und im Freundeskreis sowie durch mediale Vermittlung. Empfänger aufklärerischer Botschaften müssen diese einordnen und verarbeiten können. Daraus kann eine Entwicklung gesundheitsförderlicher Einstellungen und Verhaltensweisen erwachsen. Informationen und Gespräche über illegale Drogen im Kindesalter sind zum Beispiel in der Regel nicht angemessen. Aber wenn Kinder um sich herum den Konsum von Cannabis erleben, oft Heroinabhängigen begegnen und Spritzbestecke finden oder ihr Interesse an diesen und anderen illegalen Drogen durch die Medien stark geweckt wird, dann müssen sie auch Antworten auf ihre Fragen bekommen.

b) Klärungen ermöglichen statt belehren. Es kommt darauf an, Fragen so aufzugreifen oder aufzuwerfen, dass es zum Nachdenken, zu Gesprächen und eigenen Nachforschungen motiviert. Selbstgewonnene Erkenntnisse haben einen größeren Einfluss auf Einstellungen und Verhalten als passiv konsumierte Informationen. Besonders bewährt haben sich dabei „peer-to-peer“-Projekte und die Einbeziehung selbstkritischer KonsumentInnen. Der Austausch mit Gleichaltrigen, Gleichgestellten oder mit reflektierten Erfahrenen erhöht die Glaubwürdigkeit aufklärerischer Botschaften.

\section{Praxisbeispiele:}

„Haschisch - Gute Seiten, schlechte Seiten“ für Jugendliche kombiniert mit „Information für Eltern, Lehrer und Erzieher zu Haschisch und Marihuana“, eine ansprechend gestaltete und Gespräche stiftende Broschüre des Jugendund Drogenberatungszentrums Hannover für Jugendliche und Eltern.

- „Cannabis denn Sünde sein?“ mit dem ersten Kiffertest. Eine Broschüre rund ums Kiffen“ (1998) des Therapieladens e.V. in Berlin, weitergeführt mit der Broschüre und der Webpage „Drogen und Du“ inklusive dem selbstreflexiven Kommunikationsangebot „Check Dein Risiko“ (2001).
„Info-Cards“: Gefördert durch die Europäische Union hat das Hamburger Büro für Suchtprävention zusammen mit dem Jellinek-Zentrum in Amsterdam und Lifeline in Manchester ab 1996 mit peeredukativen Methoden geschlechtsspezifische „Info-Cards“ zu Ecstasy entwickelt und evaluiert, die auf Techno-Großveranstaltungen und in Techno-Discos von Peers verteilt werden. Die Anliegen des Projekts sind, unter EcstasykonsumentInnen Informationen über substanzspezifische Konsumrisiken zu verbreiten, dem Übergang von Probier- und Gelegenheitskonsum zu abhängigem Gebrauch vorzubeugen und Selbstkonzepte durch konsumbegleitende Beratung und Lebenshilfe zu stärken.

\section{Basisfähigkeiten fördern}

Die Vermittlung von drogenkundlichem Wissen befähigt zur Vermeidung von Konsum- und Suchtrisiken nur dann, wenn die Personen der Zielgruppe in der Lage sind, dieses Wissen in ihrem Handeln auch zu berücksichtigen. Das hängt entscheidend davon ab, ob sie über genügend Selbstvertrauen und Selbstwertgefühl verfügen, kommunizieren, soziale Kontakte herstellen und aufrechterhalten können, konfliktfähig sind, mit Entwicklungsaufgaben, Problemen und Krisen gesundheitsbewusst zurechtkommen, Konsumanimationen durchschauen und widerstehen können. Risikokompetenz setzt Kenntnisse und die beschriebenen Stärken und Fähigkeiten voraus.

Die Förderung dieser persönlichen Ressourcen ist nach wie vor als eine wesentliche Aufgabe von Suchtprävention wahrzunehmen. Persönlichkeitsförderung in diesem Sinne kann nicht erst im Jugendalter einsetzen. Sie muss bereits in der Familie und Vorschulerziehung beginnen. Gleichzeitig ist sie langfristig und kontinuierlich anzulegen. Schließlich entwickelt sich eine starke Persönlichkeit, die weiß, was gut für sie ist, nicht von heute auf morgen.

Praxisbeispiele: „Fit und stark fürs Leben“ und „Eigenständig werden“

„Fit und stark fürs Leben. Persönlichkeitsförderung zur Prävention von Aggression, Rauchen und Sucht“ lautet der Titel eines Unterrichtsprogramms, das im Rahmen des Aktionsplans „Europa gegen den Krebs“ entwickelt worden ist und vom Ernst-Klett-Grundschulverlag seit 1998 für die Klassen 1-6 in drei Heften angeboten wird. Es enthält für jeweils zwei Schuljahre 20 stimmig aufeinander aufgebaute, gut strukturierte Unterrichtsvorschläge mit einer Fülle konkreter altersangemessener Anregungen (Lieder, Entspannungsübungen, Phantasiereisen, Rollenspielvorlagen, Beobachtungs-, Reflexions- und Arbeitsaufträge) zu folgenden Schwerpunkten: Selbstwahrnehmung und Einfühlungsvermögen, Umgang mit Stress und belastenden Emotionen, Kommunikation, kritisches Denken, Standfestigkeit und Problemlösen. Es regt SchülerInnen zum selbstständigen erfahrungsbezogenen Lernen an [31]. Die hier beschriebenen Qualitätsmerkmale gelten auch für das „Unterrichtsprogramm für die Gesundheitsförderung und Suchtprävention - Eigenständig werden“, das mit Unterstützung der Stiftung Mentor-Deutschland vom In- 
stitut für Therapie- und Gesundheitsforschung in Kiel entwickelt wurde und verknüpft mit Lehrerfortbildungen an deutschen Schulen verbreitet wird.

\section{Lustvolle Alternativen zum Drogenkonsum ermöglichen}

Je langweiliger, erlebnisärmer und öder Jugendliche ihr Leben empfinden, desto attraktiver wird für sie der Gebrauch von Drogen und die Vernachlässigung von Konsumrisiken. Diesen Erkenntnissen ist mit erlebnispädagogischen Angeboten Rechnung zu tragen, die neben der Stärkung von Selbstachtung und Konfliktfähigkeit Glücksmomente durch Grenzerfahrungen, besondere Erfolge und positive Gruppenerlebnisse vermitteln. Dazu gehören Gruppenaktivitäten wie Zirkus-, Kletterwand- oder andere risikobetonte Sportprojekte, abenteuerliche Unternehmungen in der Natur („outward bound“ etc.), die sich erheblich vom normalen Alltag eines/r Jugendlichen unterscheiden, aber auch mediale, bildend künstlerische, Theater- und Musikprojekte [32]. Gemäß den Empfehlungen der BZgA-Expertise zur Primärprävention des Substanzmissbrauchs kommt es insbesondere bei Jugendlichen mit sozialen Belastungen und Problemverhalten darauf an, Alternativerfahrungen zum Drogengebrauch und suchtriskanten Verhalten zu vermitteln.

\section{Praxisbeispiel: Kölner Spielecircus}

Unter dem Motto „Starke Kinder - Gemeinsam gegen Sucht“ bietet das Team von Heiner Kötter und Dr. Harald Schmid im Rahmen der BZgA-Kampagne „Kinder stark machen“ einen schulischen Suchtpräventionstag mit Erlebnissen in der Zirkusmanege für jeweils 60-150 Schülerinnen und Schüler der Klassenstufen 6 bis 8 an. Der Suchtpräventionstag beginnt mit Informationen zur Frage „Wie können wir uns vor Sucht und Drogen schützen?" Anschließend bereiten die teilnehmenden SchülerInnen in unterschiedlichen Workshops (gruppendynamische Spiele, Trommeln und Tanz, Akrobatik und Jonglieren, Rollenspiele) jeweils einstündige Präsentationen für eine Abschlussveranstaltung vor. Die Präsentationen sollen den geladenen Eltern, Lehrkräften und MultiplikatorInnen verdeutlichen, was Kinder brauchen, um stark genug für ein Leben ohne Sucht und Drogen zu sein.

\section{Aufhebung der strikten Trennung zwischen primärer und sekundärer Suchtprävention: frühzeitige gezielte Hilfen bei problematischem Drogenkonsum}

Es wird geschätzt, dass mindestens $90 \%$ der Jugendlichen Drogengebrauch nur transitorisch während der Lebensphase Jugend („adolescence-limited“) betreiben. Bei bis zu 10\% könne jedoch davon ausgegangen werden, dass sie vor dem Hintergrund biografisch kumulierter Belastungen und eines sich schon seit früher Kindheit aufbauenden Problemverhaltens zu riskantem Drogenkonsum neigen würden [19]. Diese Risikogruppen, z.B. Kinder aus suchtbelasteten Familien oder Kinder mit sexuellen Missbrauchserfahrungen, benötigen gezieltere Ansprachen und Hilfen.

Die Drogen- und Suchtkommission beim Bundesministerium für Gesundheit empfahl daher in ihrer Stellungnahme zur Verbesserung der Suchtprävention im Juni 2002 die Aufgabe der strikten
Trennung von primären und sekundärpräventiven Maßnahmen. Bundesweit setzt sich die Erkenntnis durch, dass an der Schnittstelle von Suchtprävention zum Hilfesystem Früherkennung und Frühinterventionsmaßnahmen aufgebaut werden sollten. Das erfordert Kooperationen zwischen Schulen, außerschulischen Freizeiteinrichtungen und ambulanten Suchtberatungsstellen.

\section{Praxisbeispiel: „Bekifft in der Schule - Hilfen für Schulen zur Vorbeugung und Lösung von Problemen“}

Als Antwort auf die Zunahme jugendlichen Cannabiskonsums auch vor und während der Schulzeit hat das SuchtPräventionsZentrum der Behörde für Bildung und Sport in Hamburg zusammen mit Suchtberatungsstellen ein Qualifizierungsund Hilfsangebot für Schulen vor allem zur Früherkennung und Frühintervention entwickelt.

Wesentliche Bestandteile sind:

- Unterstützung bei der Entwicklung und Verankerung tragfähiger Regeln für den Umgang mit Drogenkonsum sowie von Maßnahmen bei Regelverletzungen;

- Training schulischen Personals zur Problemwahrnehmung und motivierenden Gesprächsführung;

Hilfe beim Aufbau und der Durchführung einer „minimalen Intervention“ für SchülerInnen, die in problematischer Weise Cannabis konsumieren. Mithilfe eines so genannten H\&M-Tests (Haschisch und Marihuana) werden die vorhandenen Kenntnisse überprüft, aufgefrischt oder korrigiert. Ein Kiffertagebuch hilft bei der kritischen Reflexion des Cannabisgebrauchs, der Klärung von Konsummotiven und -alternativen. Bei Bedarf werden weitergehende Hilfen vermittelt.

\section{Praxisbeispiel: Modellprojekt „Frühintervention bei erst- auffälligen Drogenkonsumenten (FreD)“}

Dieses an 15 Standorten in acht Bundesländern erprobte Projekt bietet erstauffälligen jungen KonsumentInnen im Alter von 14 bis 21 Jahren nach einem so genannten „intake-Gespräch“ ein möglichst freiwillig anzunehmendes achtstündiges Kursangebot zur Information/Aufklärung sowie Beratung im Hinblick auf Drogen, Gesundheits- und Rechtsfragen. Zumeist sind Polizei und Jugendgerichtshilfe die Vermittlungsinstanzen. Ziel des Angebots ist es, die Risiken einer sich anbahnenden Abhängigkeit zu reduzieren und einem Abrutschen in die Kriminalität entgegenzuwirken.

\section{Offene Fragen, Probleme und Optionen}

1. Der Begriff „Suchtprävention“ scheint zuweilen zu eng, um die Vielfalt, Differenziertheit und Integration der aktuellen präventiven und gesundheitsförderlichen Konzepte widerzuspiegeln. Es geht heute nicht nur um die Befähigung zur Vermeidung von Suchtproblemen, sondern auch von anderen Konsumrisiken. Man muss nicht AlkoholikerIn oder von Cannabis abhängig sein, um durch Alkoholkonsum bzw. Cannabisgebrauch im Straßenverkehr, in der Schule oder 
am Arbeitsplatz Probleme zu bekommen. Angesichts der Heterogenität von Maßnahmen, Konzepten und Zielen wird moniert, dass auf vielen Ebenen der Suchtprävention immer noch eine inkohärente bis verwirrende Begrifflichkeit herrsche [33]. Eine präzisere Fassung des Leitbegriffs ist auch unseres Erachtens wünschenswert, aber sicher in einer kurzen, prägnanten Formulierung schwierig und aufgrund der Etabliertheit des Begriffes wahrscheinlich kaum einzuführen.

2. Die Praxis der Suchtprävention setzt nach wie vor zumeist auf der Verhaltensebene an. Die gesellschaftlichen Verhältnisse mit ihren Suchtprävention erschwerenden Strukturen werden zu wenig berücksichtigt. Das betrifft verschiedene Aspekte:

- Angesichts der Werbeausgaben für Alkohol, Tabak und Psychopharmaka sowie der Grundbotschaft vieler Werbungen „Bedürfnisbefriedigung durch Konsum, und zwar sofort!“ ist es schwierig, dagegen suchtpräventive Orientierungen für den Umgang mit Suchtmitteln zu vermitteln. Ein Konsumparadigma ist für die Suchtprävention schädlich. Hilfreich wären Werbeverbote für die legalen Drogen, um die allgegenwärtigen Konsumanimationen für diese Substanzen wesentlich zu reduzieren. Den Mut zu diesem Schritt und die dafür nötige Konfliktbereitschaft muss die Drogenpolitik aufbringen. Tabakwerbung erhöht die Anfälligkeit von Jugendlichen, mit dem Rauchen zu beginnen. Länder mit Werbeverboten für Tabakwaren (Finnland, Frankreich, Neuseeland und Norwegen) weisen eine erheblich höhere Reduzierung des Konsums auf als Deutschland [34].

- Von vielen Menschen wird es als doppelmoralisch empfunden, dass die gesetzlichen Maßnahmen zur Einschränkung des Konsums einiger Drogen in keinem ausreichend (toxiko)logischen Zusammenhang mit ihrer psychischen und physischen Schädlichkeit stehen, gleichzeitig die Vermarktung von Alkoholika und deren Konsum fast grenzenlos toleriert wird. Da der Erfolg suchtpräventiver Bemühungen nicht zuletzt von der Glaubwürdigkeit ihrer Botschaften abhängt, benötigen wir unbedingt eine drogenpolitische Klärung, wie zukünftig mit legalen und illegalen Drogen umgegangen werden soll. Ist es zu verantworten, dass an Tankstellen und Autobahnraststätten Alkoholika verkauft werden dürfen? Was muss geschehen, damit Jugendschutz ernster genommen wird? Das betrifft den Verkauf von Alkoholika und „Alcopops“ an Kinder und Jugendliche unter 16 Jahren im Widerspruch zu den geltenden gesetzlichen Bestimmungen sowie die weltweit höchste Dichte von Zigarettenautomaten in der Bundesrepublik Deutschland - zumeist in Reichweite auch für Kinderhände. Könnte die Entkriminalisierung des Erwerbs und Besitzes von Cannabis und anderen Substanzen zum Eigenverbrauch nicht ein wichtiger Schritt auch im Sinne von Suchtprävention sein, um die Attraktivität des Verbotenen aufzuheben, offene Kommunikation zu ermöglichen und in diesem Klima Risikokompetenzen zu fördern?

- Was kann die Förderung von Selbstachtung und Konfliktfähigkeit im hier beschriebenen Sinne erreichen, wenn belastende Lebensbedingungen und -ereignisse ihre Entwicklung und Anwendung gravierend behindern oder sogar nicht zulassen? Suchtprävention muss sich deshalb grundsätzlich für die Verbesserung solcher Verhältnisse einsetzen. Strukturelle Bedingungen wie Arbeitsplätze, Lehrstellen und ausreichende Jugendfreizeitangebote stellen nach [13] die „wesentlichen Grundpfeiler“ erfolgreicher primärer Suchtprävention dar.

3. Bei ihrem sinnvollen Bemühen um Erlebnisalternativen zum Drogengebrauch sollte die Suchtprävention nicht jedem Konsumtrend der Erlebnisgesellschaft hinterherhecheln und mit einer Überdosis pädagogischer Angebote Kindern und Jugendlichen die Luft für eigene Phantasien und Aktivitäten nehmen. Es ist aus suchtpräventiver Sicht zu begrüßen, wenn Kinder und Jugendliche sich mit allen Sinnen und schöpferischem Eigensinn ihre scheinbar banalen Lebenswelten auf aktive, abenteuerliche Weise soweit wie möglich selbst nur erschließen und gestalten. Ein Leben im Stil des Huckleberry Finn, so bilanziert [35] therapeutische Erfahrungen, schützt vor Suchtrisiken.

4. In der praktischen Umsetzung des Konzeptes der Förderung von Risikokompetenz tun sich Probleme auf. Die unterschiedlichen Gefährlichkeiten der Substanzen müssen genauso bedacht werden wie die unterschiedlichen Voraussetzungen verschiedener Zielgruppen. Feldversuche hinsichtlich des Umgangs mit der gesellschaftsfähigen Droge Alkohol lassen sich wohl am ehesten verantworten. Abgesehen davon ist zu betonen, dass suchtpräventive Angebote zur Förderung der Risikokompetenz nicht für jede/n Heranwachsenden oder Erwachsenen gleichermaßen geeignet sind. Infrage kommen nur Zielgruppen, deren Lebenslagen, Entwicklungsvoraussetzungen und Lebensstile Potenziale zur Ausprägung dieser Kompetenz vermuten lassen, die im Übrigen immer auch Abstinenz als Verhaltensoption mit enthält.

Safer-Use-Aufklärungen sollten sich nicht an Jugendliche wenden, die die jeweils angesprochenen Drogen noch gar nicht konsumieren. Das könnte zum Konsum animieren und sich damit kontraproduktiv für Suchtprävention auswirken. Ein vorverlagerter Konsumbeginn durch zu frühe explizit risikobezogene Informationen z.B. wäre schädlich, ja fatal. Für Alkohol und Partydrogen liegen eindeutige Anzeichen dafür vor, dass ein zu früher Experimentierund Konsumbeginn die Möglichkeit der Kompetenzentwicklung, der Transitionierung und der Selbstkontrolle in späteren Jahren massiv behindert, wenn nicht unmöglich macht.

Aus primärpräventiver Sicht ist auch zu fragen, ob eine Vielzahl der Heranwachsenden nicht prinzipiell mit Angeboten zur Förderung von Risikokompetenz überfordert ist, ob ihnen durch diese „Modernisierung“ der Suchtprävention der Prozess der Sozialisierung, Ressourcenbildung und Entwicklung von Selbstwirksamkeit nicht sogar noch erschwert würde. Vor dem Hintergrund massiver sozialer Ungleichheiten sowie neuer sozialökologischer Ungleichbehandlungen wäre es naiv, der Suchtprävention zu unterstellen, sie vermöge durch ihre Interventionen, Chancengleichheit im Kompetenzerwerb zu erzeugen. Für Informationsaufnahme wie für praktisches Risikomanagement, für individuelle Selbstkontrolle und pädagogische Risikobegleitung gibt es immer gut oder zumindest mittelmäßig zugängliche, genauso wie weitgehend unzugängliche Gruppen und Lebenslagen („hard to reach“). Letztere sind in der Regel am Grad ihrer sozialen Belastung, an Bildungsdefiziten und einem Status als „Modernisierungsverlierer“ zu erkennen. 
Unklar ist trotz erheblicher Anstrengungen in der aktuellen Sozialisations- und Jugendrisikoforschung weiterhin, welchen Umfang diese Gruppen haben und welcher Veränderungsdynamik sie unterliegen. Für die Minderheit der bereits vor dem Jugendalter auffälligen Kinder heißt Suchtprävention vorrangig Früherkennung in Verbindung mit erzieherischer, sozialpädagogischer oder therapeutischer Einflussnahme auf die Verhaltensstörungen [36]. Angebote dafür gilt es verstärkt in pädagogischen Institutionen sowie in Kinder-, Jugend- und Hausarztpraxen aufzubauen [37]. Denn Lebenskompetenzförderung mag auch für Risikojugendliche nützliche Wirkungen beim Übergang ins Jugendalter entfalten. Sie wirkt aber nicht auf die grundlegenden, die nach Silbereisen „eigentlichen“ Risikofaktoren dieser Gruppe.

Präventiv umsetzbare Handreichungen auf der Grundlage allgemeiner wie risikobezogener entwicklungswissenschaftlicher Forschung fehlen besonders für die Altersphase von 10 bis 13/14 Jahren. Über die Realisierungsmöglichkeiten abgestufter Interventionen zur Begleitung von Risikoerfahrungen liegen etliche subjektive Theorien und private Einzelfallmodelle vor, es gibt aber bei weitem zu wenige wissenschaftlich begründete Projektauswertungen oder übertragbare Ergebnisse aus der empirischen Wirkungsforschung. Das einer verengten Forschungstradition anzulastende Mangelwissen über gelungene Risikokarrieren und über erfolgreich bestandene drogenbezogene Transitionsprozesse in der Jugend und in späteren Lebensphasen bleibt zu beklagen.

\section{Literatur}

${ }^{1}$ Nöcker G. Suchtprävention in Deutschland. In: Deutsche Hauptstelle gegen die Suchtgefahren e.V (Hrsg). Jahrbuch Sucht 2000. Hamburg: Neuland, 2000; 177-194

${ }^{2}$ Hüsgen HA. Drogenpolitik und Drogenprävention in der Entwicklung. Prävention 2003; 26 (1): $13-17$

${ }^{3}$ Barth J, Bengel J. Prävention durch Angst? Stand der Furchtappellforschung. Köln: BZgA (Forschung und Praxis der Gesundheitsförderung, Band 4), 1996

${ }^{4}$ Antonovsky A. Salutogenese. Zur Entmystifizierung der Gesundheit. Tübingen: dgvt, 1997

${ }^{5}$ Bengel J, Strittmatter R, Willmann H. Was erhält Menschen gesund? Antonovskys Modell der Salutogenese. Köln: BZgA (Forschung und Praxis der Gesundheitsförderung, Band 6), 1998

${ }^{6}$ BZgA Bundeszentrale für gesundheitliche Aufklärung (Hrsg). Leitbegriffe der Gesundheitsförderung. Schwabenheim: Sabo, 2003

${ }^{7}$ Jessor RL, Jessor S. Die Entwicklung Jugendlicher und der Beginn des Alkoholkonsums. In: Vogler RE, Revenstorf D (Hrsg). Alkoholmissbrauch. München: Urban \& Schwarzenberg, 1978; 21 - 44

${ }^{8}$ Silbereisen RK, Kastner P. Jugend und Drogen: Entwicklung von Drogengebrauch - Drogengebrauch als Entwicklung? In: Oerter R (Hrsg). Lebensbewältigung im Jugendalter. Weinheim: VCH, 1985; 192 - 219

${ }^{9}$ Franzkowiak P. Risikoverhalten und Gesundheitsbewußtsein von Jugendlichen. Berlin/Heidelberg: Springer, 1986

${ }^{10}$ Nordlohne E. Die Kosten jugendlicher Problembewältigung. Weinheim/München: Juventa, 1992

${ }^{11}$ Engel U, Hurrelmann K. Was Jugendliche wagen. Weinheim/München: Juventa, 1993

12 Künzel-Böhmer J, Bühringer G, Janik-Konecny T. Expertise zur Primärprävention des Substanzmissbrauchs. Baden-Baden: Nomos, 1993
${ }^{13}$ Denis A et al. Fortschreibung der Expertise zur Primärprävention des Substanzmissbrauchs. Köln/München: BZgA/IFT, 1994

${ }^{14}$ Püschl M. Aufklärung und Konsumberatung - oder was sich in der Suchtprävention durch Ecstasy verändert hat. In: Rabes M, Harm W (Hrsg). XTC und XXL Ecstasy - Wirkungen, Risiken, Vorbeugungsmöglichkeiten und Jugendkultur. Reinbek bei Hamburg: Rowohlt, 1997; $201-211$

${ }^{15}$ Püschl M, Schlömer H. Von der Abschreckungspädagogik zur Förderung von Risikokompetenz - die Entwicklung der Suchtprävention am Beispiel Hamburgs. In: Krausz M, Raschke P (Hrsg). Drogen in der Metropole. Freiburg: Lambertus, 1999

${ }^{16}$ Rabes M, Harm W (Hrsg). XTC und XXL Ecstasy - Wirkungen, Risiken, Vorbeugungsmöglichkeiten und Jugendkultur. Reinbek bei Hamburg: Rowohlt, 1997

17 Stöver H (Hrsg). Akzeptierende Drogenarbeit - Eine Zwischenbilanz. Freiburg: Lambertus, 1999

${ }^{18}$ Heudtlass JH, Stöver H (Hrsg). Risiko mindern beim Drogengebrauch. 2. neu bearb. Auflage. Frankfurt/M: Fachhochschulverlag, 2000

${ }^{19}$ Silbereisen RK. Entwicklungspsychologische Aspekte von Alkoholund Drogengebrauch. In: Oerter R, Montada L (Hrsg). Entwicklungspsychologie. Weinheim: Beltz PVU, 1995; 1056-1068

20 Shedler J, Block J. Adolescent Drug Use and Psychological Health. A longitudinal inquiry. American Psychologist 1990; 45 (5): 612 - 630

${ }^{21}$ Franzkowiak P. Risikokompetenz - Eine neue Leitorientierung für die primäre Suchtprävention? Neue Praxis 1996; 26 (5): 409-425

22 Franzkowiak P. Risikokompetenz und 'Regeln für Räusche' - Was hat die Suchtprävention von der akzeptierenden Drogenarbeit gelernt? In: Stöver H (Hrsg). Akzeptierende Drogenarbeit - Eine Zwischenbilanz. Freiburg: Lambertus, 1999; 57-73

${ }^{23}$ Silbereisen RK, Reese A. Substanzgebrauch: Illegale Drogen und Alkohol. In: Raithel J (Hrsg). Risikoverhaltensweisen Jugendlicher. Opladen: Leske \& Budrich, 2001; 131 - 153

${ }^{24}$ Fahrenkrug H. Risikokompetenz - eine neue Leitlinie für den Umgang mit 'riskanten Räuschen'? Suchtmagazin 1998; 24 (3): 23 - 27

${ }^{25}$ Frietsch R. Grundsätzliches zur (Sucht-)Prävention. Sozialmagazin 1994; 19 (5): $14-19$

${ }^{26}$ Gantner A. Check Dein Risiko - Ein selbstreflexives Kommunikationsangebot in der Sekundärprävention. Prävention 2001; 24 (4): 116 - 118

27 Püschl M, Schlömer H. Suchtprävention 2002. In: Böllinger L, Stöver H (Hrsg). Drogenpraxis Drogenrecht Drogenpolitik. Frankfurt/M: Fachhochschulverlag, 2002

${ }^{28}$ Schwarting F. Zwischen Wunsch und Wirklichkeit - Zum alltäglichen Umgang mit Alkohol konsumierenden Mädchen und Jungen in Einrichtungen der Jugendhilfe. In: Amt für Jugend/Büro für Suchtprävention (Hrsg). Alkohol - Irgendwann ist genug. Hamburg: Amt für Jugend/Büro für Suchtprävention, 2000; 13-21

${ }^{29}$ Wiborg G, Hanewinkel R. Be Smart - Don't Start - Der Nichtraucherwettbewerb in Deutschland. Abschlussbericht. Kiel: IFT-Nord, 1997

${ }^{30}$ Hanewinkel R, Wiborg G. Primär- und Sekundärprävention des Rauchens im Jugendalter - Effekte der Kampagne „Be Smart - Don't Start“. Gesundheitswesen 2002; 64 (8/9): $494-498$

${ }^{31}$ Burow F et al. Fit und stark fürs Leben. Stuttgart: Klett, $1998 \mathrm{ff}$

${ }^{32}$ Hallmann HJ. Suchtprävention als Kompetenzbildung - Grundlagen erlebnisorientierter Suchtvorbeugung in der Jugendarbeit. In: Krätzschmar L, Krauss G (Hrsg). Schaden verhindern - Perspektiven eröffnen. Nürnberg: ISKA, 1994; 19-31

33 Hafen M. Die Begrifflichkeit in der Prävention - Verwirrung auf allen Ebenen. Abhängigkeiten 2001; 7 (1): 33-49

${ }^{34}$ Hanewinkel R, Pohl J. Werbung und Tabakkonsum. Kiel: IFT, 1988

${ }^{35}$ Schiffer E. Warum Huckleberry Finn nicht süchtig wurde. Weinheim/ Berlin: Beltz Quadriga, 1993

${ }^{36}$ Silbereisen RK. Differenzierungen und Perspektiven für Prävention aus entwicklungspsychologischer Sicht. In: Kolip P (Hrsg). Programme gegen Sucht. Weinheim/München: Juventa, 1999; 70-85

37 Fiedler A, Kremer G. Suchtvorbeugung und Frühintervention in der medizinischen Praxis. Prävention 2001; 24 (4): 122-124 